\title{
The Effect of Expanded Measurement Uncertainty on the Outcome of Blood Pressure Measurement Validation Protocol Based on the ISO81060-2:2018 Guideline - A Monte Carlo Simulation Approach
}

\author{
Janos Palhalmi ${ }^{1}$ \\ ${ }^{1}$ DataSenseLabs Ltd, Budapest, Hungary
}

\begin{abstract}
The most common hemodynamic measurement technologies are the classical auscultatoric sphygmomanometric method and numerous solutions for automated sphygmomanometry. The classical method is the reference for testing the automated solutions according to the ISO81060-2:2018 guideline.

Both of the above mentioned blood pressure estimations are indirect measurement methods according to the approach of metrology.

According to the ISO81060-2:2018 guideline, two indirect measurement and estimation methods are compared with each other without the theoretical and technical consideration of the possible effect of the measurement uncertainty on the results.

Within this simulation study several components of the measurement uncertainty were implemented to test the reliability and confidence limit of the ISO guideline recommended process flow, experimental design and statistical analysis.
\end{abstract}

\section{Introduction}

In the field of medical technology and health informatics more and more wearable solutions appear on the market which can be capable of medical grade cardiac remote monitoring.

On the other hand / in contrary, the standardization of wearable biosensors capable of hemodynamic remote monitoring is a more complex task both from manufacturing design and form the testing and validation perspective.

The diagnosis and the treatment plans of blood pressure diseases are strictly based on repetitive measurements where the accuracy and reliability of the home measurement device is a crucial factor.

Based on population statistics of blood pressure values, the frequency of the false positive/negative diagnosis of hypertension could be decreased approximately by $20 \%$ if the systematic errors of systolic and diastolic blood pressure measurements were less than 1 and $3 \mathrm{Hgmm}$ respectively $[1,2,3]$.

The test process flow and the validation protocol of the newly developed automated sphygmomanometers is summarized in the ISO81060-2:2018 guideline [4]. Development and specification of this ISO guideline is continuously in progress, since many consensus documents have been published recently to specify the disturbing and influencing factors of the measurement process and to define the problem of downsampling $[5,6]$.

Maximum permissible error of the reference method (analog/aneroid sphygmomanometer) is a defined condition in validation protocols, but the effect of the expanded measurement uncertainty is not considered in the validation statistics.

The aim of this study was to compare the outcome of the statistical classification with and without the consideration of the expanded uncertainty of the blood pressure measurement. In the simulations special attention was paid to explore the effect of the asymmetric averaging within the process (1.1. and 2.1. on Fig.1.) on the results of the comparative analysis.

\section{Methods}

Components of the standard measurement uncertainty were determined based on the relevant OIML standards [7, $8,9]$, ISO guidelines [4] and data available in the literature $[10,11]$. Components of standard measurement uncertainty related to random error, systematic error and maximum permissible error were combined according to formula 1 .

Calculation of the standard measurement uncertainty:

\section{Formula 1.}

$$
\mathrm{u}_{\mathrm{c}}(\mathrm{x})=\sqrt{\sum_{\mathrm{i}=1}^{\mathrm{n}}\left(\mathrm{u}\left(\mathrm{x}_{\mathrm{i}}\right)\right)^{2}}
$$

$\mathrm{u}_{\mathrm{c}}(\mathrm{x})$ : standard (combined) measurement uncertainty $\mathrm{u}\left(\mathrm{x}_{\mathrm{i}}\right)$ : ith components of the standard measurement 
uncertainty related to random error, systematic error and maximal permissible error (see Table 1.) $\mathrm{n}=7$ for reference device, $\mathrm{n}=5$ for the device under test.

$$
\mathrm{U}=\mathrm{k} * \mathrm{u}_{\mathrm{c}}(\mathrm{x}) ; \mathrm{k}=2
$$

\section{Formula 2.}

$\mathrm{U}$ : expanded measurement uncertainty

$\mathrm{u}_{\mathrm{c}}(\mathrm{x})$ : standard (combined) measurement uncertainty

In this work, components of the measurement uncertainty originated from the computational algorithms implemented into the automatic devices under test were not considered because these are negligible [12] compared with the uncertainty components originated from the ISO process flow (Fig.1.).

\section{Results}

Following the extensive analysis of the validation protocols [7, 8, 9, 10, 11] and the ISO81060-2:2018 guideline, seven uncertainty components were defined to compute the expanded uncertainty (U) for of the systolic BP measurement.

\begin{tabular}{|c|c|c|}
\hline $\begin{array}{l}\text { Sources of measurement } \\
\text { uncertainty components }\left(u_{(x)}:\right)\end{array}$ & $\begin{array}{l}\text { Reference } \\
\text { Manometer } \\
\text { (Mercury } \\
\text { mm) }\end{array}$ & $\begin{array}{l}\text { Tested device } \\
\text { (Mercury } \\
\mathrm{mm})\end{array}$ \\
\hline $\begin{array}{l}u_{l(x):} \text { Maximal permissible } \\
\text { error (static) }\end{array}$ & 1 & 1.5 \\
\hline $\begin{array}{l}u_{2(x)}: \text { Maximal permissible } \\
\text { error (dynamic) }\end{array}$ & 1 & 2.5 \\
\hline$u_{3(x)}:$ Analog scale $\ldots$ error & 0.4 & 0 \\
\hline$u_{4(x)}:$ Terminal digit bias & 1.06 & 0 \\
\hline$u_{5(x)}:$ Cuff pressure speed bias & 0.5 & 0 \\
\hline $\begin{array}{l}u_{6(x)}: \text { Biological variability } \\
\text { caused bias }\end{array}$ & 0.76 & 0.76 \\
\hline $\begin{array}{l}u_{7(x)} \text { : Bias caused by the human } \\
\text { subject's behaviour }\end{array}$ & 0.4 & 0.4 \\
\hline $\begin{array}{l}u_{(c)}: \text { Standard measurement } \\
\text { uncertainty }\end{array}$ & 2.07 & 3.04 \\
\hline $\begin{array}{l}\text { U: Expanded measurement } \\
\text { uncertainty }\end{array}$ & \pm 4.14 & \pm 6.08 \\
\hline
\end{tabular}

\section{Table 1.}

Table summarizes the components and sources of the blood pressure measurement uncertainty implemented into the Monte Carlo simulations.

Based on the analysis of the process flow and experimental design recommended by the ISO810602:2018 guideline, the following components were implemented into the Monte Carlo simulations (Fig. 1.):

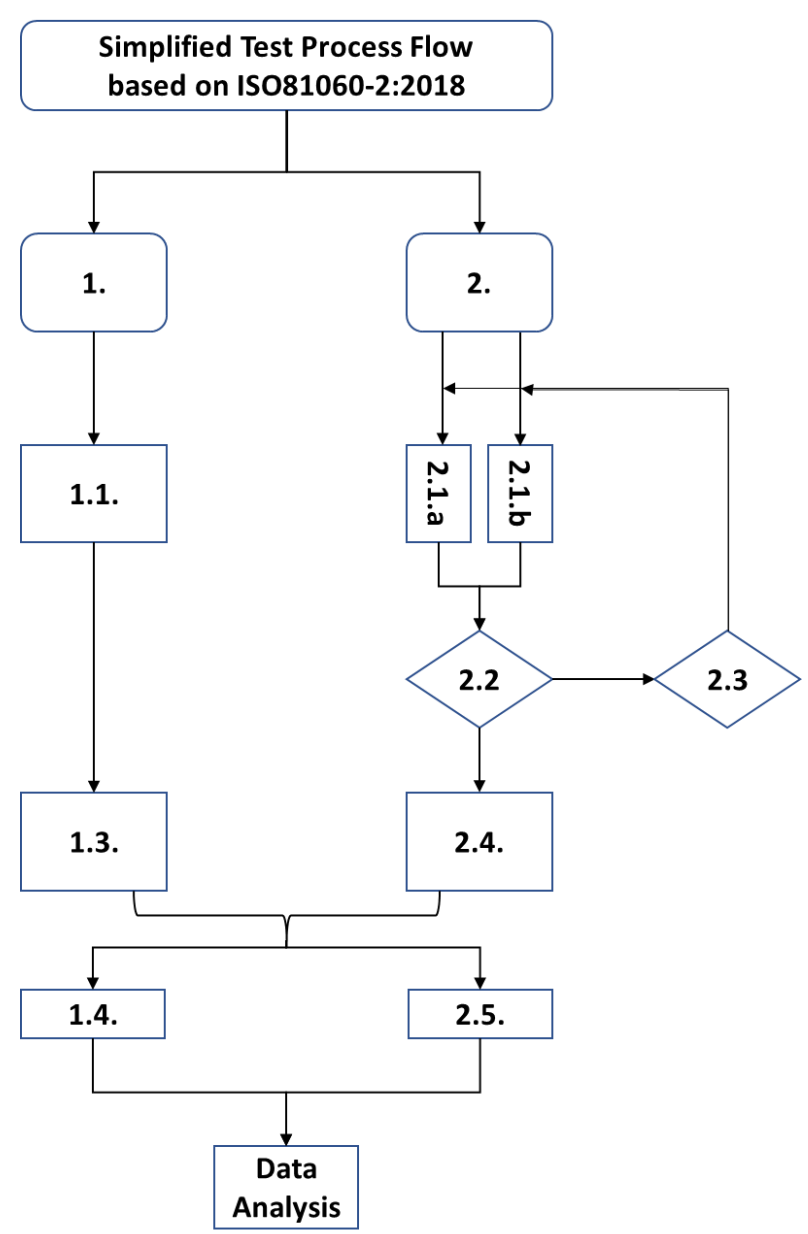

Fig.1.

Terminology of the simplified test process flow:

1. Device under test

2. Reference Sphygmomanometer

1.1. Estimated value (Hgmm) by the tested device

2.1.a. Measured value (Hgmm) by reference sphygmomanometer carried out by observer 1

2.1.b. Measured value (Hgmm) by reference sphygmomanometer carried out by observer 2

2.2. Decision making point:

ISO81060-2:2018 5.2.3.c. and 5.2.4.1.1.n., o.,1,2

\subsection{Decision output}

1.3. Mean of the estimated values (Hgmm) measured by the tested device

2.4. Mean of the measured values determined by the observers: ISO81060-2:2018 5.2.3.d.

1.4. Differences between the values of paired measurements by the tested device and the reference sphygmomanometer used by the observers.

2.5. Mean of the values of paired measurements by the tested device and the reference sphygmomanometer used by the observers.

> Data Analysis: according to ISO81060-2:2018 5.2.4.1.2.

Please note the asymmetry within the process regarding 
at the 1.1. and 2.1. subparts.

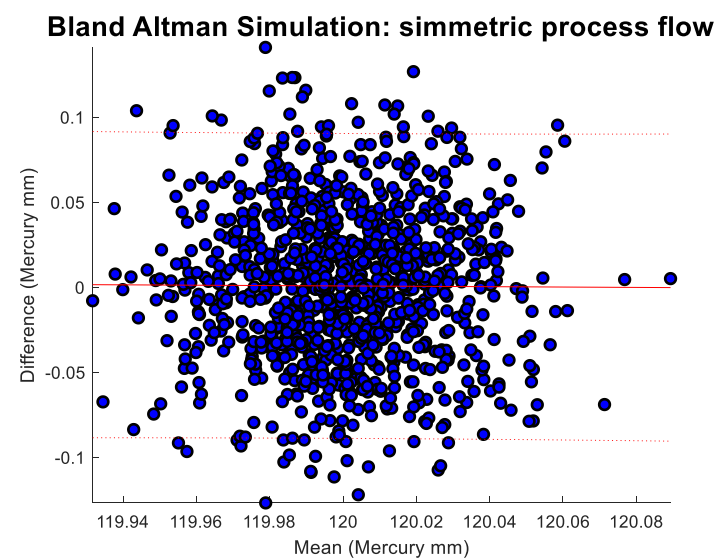

Fig. 2.A.

\begin{tabular}{|l|r|r|r|r|}
\hline & Estimate & SE & \multicolumn{1}{l|}{ tStat } & pValue \\
\hline Intercept & 1.302477 & 7.504099 & 0.173569 & 0.86224 \\
\hline Constant & -0.01085 & 0.062534 & -0.17347 & 0.86232 \\
\hline
\end{tabular}

Table 2.A.

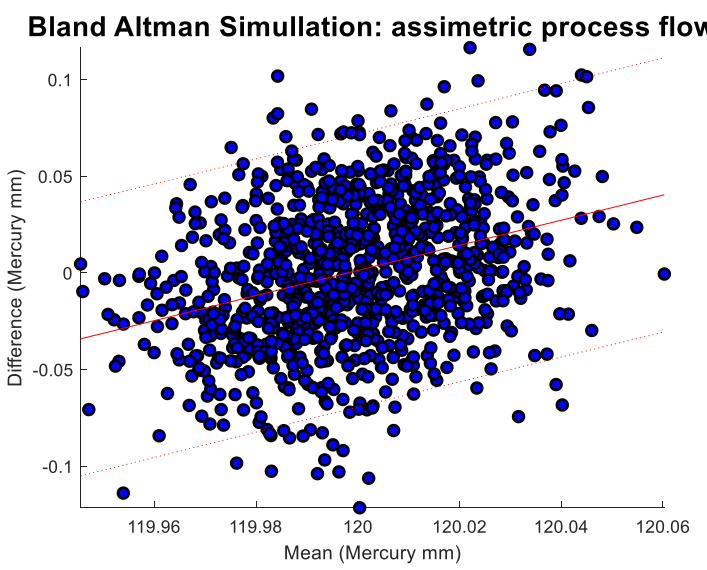

Fig. 2.B.

\begin{tabular}{|l|r|r|r|r|}
\hline & Estimate & SE & \multicolumn{1}{l|}{ tStat } & \multicolumn{1}{l|}{ pValue } \\
\hline Intercept & -77.8529 & 7.115011 & -10.9421 & $2.14 \mathrm{E}-26$ \\
\hline Constant & 0.648785 & 0.059292 & 10.94221 & $2.14 \mathrm{E}-26$ \\
\hline
\end{tabular}

Table 2.B.

Fig. 2. And Table 2.

Simulation conditions: standard normal distribution, coverage probability: $95 \%$, mean $=120$, sigma $=1$ both for reference and for the tested device.

Bland-Altman analysis of the simulated results revealed the linear bias induced by the asymmetric process flow (1.1. versus 2.1. on Fig.1.). Simulations were carried out with (Fig. 2.B. and Table 2.B.) and without (Fig. 2.A. and Table 2.A.) averaging the observers' values (Fig.1. 2.1.a. and 2.1.b.). Bias is represented by the results (Table 2. and Table 3.) of the linear regression models under each simulation conditions. Regression line: continuous red line. Confidence interval of the regression at 95 percentage of coverage probability: dotted red line.

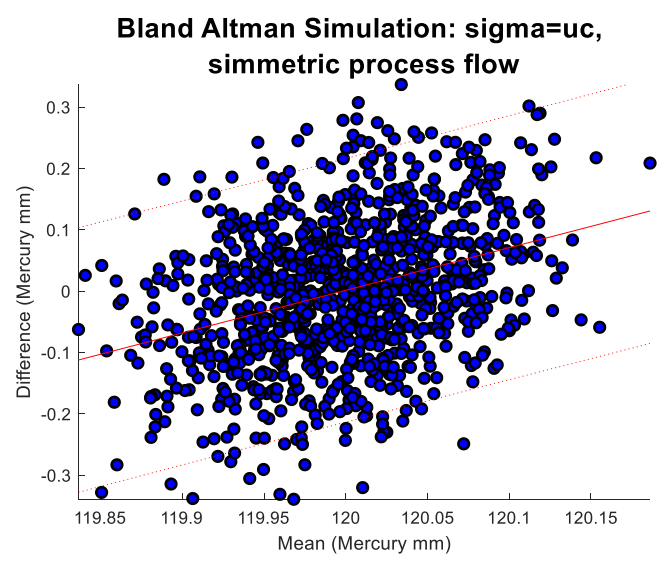

Fig. 3.A.

\begin{tabular}{|l|r|r|r|r|}
\hline & Estimate & SE & \multicolumn{1}{l|}{ tStat } & \multicolumn{1}{l|}{ pValue } \\
\hline Intercept & -83.331 & 7.185409 & -11.5973 & $2.91 \mathrm{E}-29$ \\
\hline Constant & 0.694437 & 0.059879 & 11.59726 & $2.91 \mathrm{E}-29$ \\
\hline
\end{tabular}

Table 3.A.

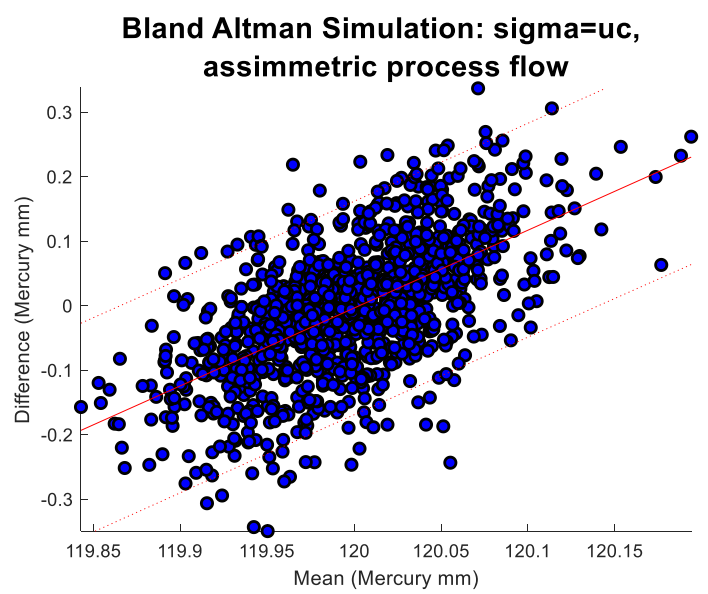

Fig. 3.B.

\begin{tabular}{|l|r|r|r|r|}
\hline & Estimate & SE & tStat & \multicolumn{1}{l|}{ pValue } \\
\hline Intercept & -144.588 & 5.957792 & -24.2688 & $1.25 \mathrm{E}-102$ \\
\hline Constant & 1.204873 & 0.049649 & 24.26778 & $1.27 \mathrm{E}-102$ \\
\hline
\end{tabular}

Table 3.B.

Fig. 3. and Table 3.

Simulation conditions: standard normal distribution, coverage probability: $95 \%$, mean $=120$, sigma $=\boldsymbol{u}_{(\boldsymbol{c})}$ for reference and for the tested device, respectively.

Bland-Altman analysis of the simulated results revealed the linear bias induced by the asymmetric process flow (1.1. versus 2.1. on Fig.1.). Simulations were carried out with (Fig. 3.B. and Table 3.B.) and without (Fig. 3.A. and 
Table 3.A.) averaging the observers' values (Fig.1. 2.1.a. and 2.1.b.). Bias is represented by the results (Table 2. And Table 3.) of the linear regression models under each simulation conditions. Regression line: continuous red line. Confidence interval of the regression at 95 percentage of coverage probability: dotted red line.

In the next section of the study, the systolic BP values measured by a reference sphygmomanometer and by a device under test $(n=304)$ from a shared database [13] were modeled by Monte Carlo simulation and were resampled $(\mathrm{m}=1000)$ with and without expanding the sampled values with the measurement uncertainty under $95 \%$ coverage probability.

According to the Bland-Altman statistical protocol, the confidence interval for the mean differences between the reference and the observed values was reconstructed at the level of $95 \%$ coverage probability: $\pm \mathrm{CI}=\sqrt{ }_{\text {sigma }^{2}} / \mathrm{n}$. The number of reliable comparisons was defined as the number of differences within the confidence interval: DiffCI.

The number of DiffCI events obtained without measurement uncertainty calculation for the reference and for the tested systolic BP values was 203 out of 304 .

The mean and standard deviation of DiffCI events obtained by Monte Carlo simulation for systolic BP values as random variables expanding only the reference values and expanding both the reference and the tested values with measurement uncertainty calculation were as follows: DiffCI mean=150.90, DiffCI $\mathrm{std}=6.65$, DiffCI mean $=129.75$, DiffCI std=7.88.

\section{Conclusions}

Although only one asymmetric component of the simplified process flow was implemented into the Monte Carlo simulations, still, a significant bias was observed during the comparison of the tested and reference values.

Including the concept of the measurement uncertainty in the comparative validation process of noninvasive blood pressure measurement methods significantly changes the number of reliable comparisons. This highlights the importance of the direct bio-signal metrology approach [14] compared to the general biostatistical approach based on indirect measurement.

\section{References}

[1] B. A. Rodrigues Filho et al. "The role of the measurement uncertainty and error in the blood pressure measurement" XXII World Congress of the International Measurement Confederation (IMEKO 2018). IOP Conf. Series: Journal of Physics: Conf. Series 1065 (2018) 132002 IOP Publishing doi:10.1088/1742-6596/1065/13/132002
[2] Eoin O'Brien et al. "The quest accuracy of blood pressure measuring devices" Journal of Clinical Hypertension. 2018; 20:1092-1095.

[3] Bhaskar Shahbabu et al. "Which is more accurate in measuring the blood pressure? A digital or an aneroid sphygmomanometer" Journal of Clinical and Diagnostic Research. 2016 Mar, Vol-10(3): LC11-LC14

[4] ISO81060-2:2013 and 2018. https://www.iso.org/standard/73339.html

[5] Stergiou et al. "A universal standard for the validation of blood pressure measuring devices: Association for the Advancement of Medical Instrumentation/European Society of Hypertension/International Organization for Standardization (AAMI/ESH/ISO) collaboration statement". Hypertension. Vol. 71:368-374. 2018

[6] G. Stergiou et al. "A universal standard for the validation of blood pressure measuring devices: Association for the Advancement of Medical Instrumentation/European Society of Hypertension/International Organization for Standardization (AAMI/ESH/ISO) collaboration statement." Hypertension. Vol. 73:e35-e66. 2019

[7] International Organization of Legal Metrology. Revision of R16-1: non-invasive non-automated sphygmomanometers. OIML R16-1 2002.

[8] International Organization of Legal Metrology. Revision of R16-2: non-invasive automated sphygmomanometers. OIML R16-2 2002.

[9] Célio H. M. Fraga et al. "A more effective approach to the legal metrological control of sphygmo - manometers." OIML Bulletin. Vol. LVIII. No. 4. 2017 October.

[10] R. N. Morcos et al. "Sources of error in office blood pressure measurement. JABFM Vol. 32. No. 5. 2019.

[11] B. J. Powers et al. "Measuring blood pressure for decision making and quality reporting: where and how many measures?" Annals of Internal Medicine. Vol. 154. No. 12. 2011.

[12] Soojeoung Lee et al., "Uncertainty in blood pressure measurement estimated using ensemble-based recursive methodology" Sensors, 2020, 20, 2108

[13] B.G.Celler et al. Novel methods of testing and calibration of oscillometric blood pressure monitors. PLOS ONE https://doi.org/10.1371/journal.pone.0201123 August 6, 2018.

[14] János Pálhalmi, Patented biosignal metrology method: ID: P1900302. https://www.sztnh.gov.hu/hu 2019.

\author{
Address for correspondence: \\ János Pálhalmi \\ Király u. 80. Budapest 1068 Hungary \\ E-mail address: janos.palhalmi@datasenselabs.net
}

